\title{
Funcionalidad para actividades de la vida diaria en adultos mayores rurales de Cárdenas, Tabasco, México
}

\author{
Functionality for daily living activities in rural elderly from Cardenas, \\ Tabasco, Mexico
}

Eduardo Fócil-Némiga', Marco Antonio Zavala-González²

\section{Resumen}

Objetivo: Evaluar la funcionalidad para las actividades de la vida diaria de los adultos mayores residentes del Poblado "General Vicente Guerrero" (C-29) de Cárdenas, Tabasco, México, en el 2011.

Material y métodos: Diseño: transversal. Universo: 194 adultos mayores. Muestra: probabilística simple, $n=113,5 \approx 114$. Muestreo: aleatorio sistematizado, $k=1,7 \approx$ 2. Criterios de selección: edad $\geq 60$ años, cualquier sexo, sin discapacidades físicas, que aceptaron participar en el estudio. Variables: edad, sexo, escolaridad, estado civil, ocupación, actividades de tiempo libre y ocio, morbilidad, control en primer nivel de enfermedades crónicodegenerativas, hospitalizaciones, funcionalidad para actividades básicas de la vida diaria (ABVD), funcionalidad para actividades instrumentales de la vida diaria (AIVD), funcionalidad para actividades avanzadas de la vida diaria (AAVD). Instrumentos: escala de Katz, índice de Lawton \& Brody, escala física de Siu y Reuben. Análisis: estadística descriptiva y razón de posibilidades (OR) con 95\% de confianza ( $p \leq 0.05$ ). Software: Epilnfo ${ }^{\circledR}$ versión 3.5.1 para entorno Windows (C).

Resultados: 114 adultos mayores, edad media 70.2 \pm 7.3 años, intervalo 60-96 años. Masculinos 54\%, femeninos $46 \%$. Portadores de enfermedad $77 \%$. Dependencia funcional: $15 \%$ básica, $66 \%$ instrumental, $68 \%$ avanzada. Factores asociados a dependencia funcional básica: edad > expectativa de vida nacional $O R=4,3\left(I_{95} 1,45,12,73\right)$, cambio de rol social $O R=6,32\left(I_{95} 1,67,23.91\right)$. Factor asociado a dependencia funcional instrumental: sexo femenino $O R=4,73\left(\operatorname{IC}_{95} 1,97,11,35\right)$. Factor asociado a dependencia funcional avanzada: sexo femenino $O R=7,58$ $\left(\mathrm{IC}_{95} 2,83,20,34\right)$.
Conclusiones: La prevalencia de dependencia para ABVD fue baja, mientras que para AIVD y AAVD fue alta. Se requieren acciones rehabilitadoras y programas de detección oportuna focalizados.

Palabras clave: (DeCS BIREME): Adulto mayor (anciano), actividades cotidianas, discapacidad, funcionalidad, independencia

\section{Abstract}

Objective: To assess functionality for daily living activities in rural elderly from "General Vicente Guerrero" village (C-29) of Cardenas, Tabasco, Mexico, in 2011.

Material and methods: cross-sectional design. Universe: 194 elderly people. Sample: simple randomized, $n=113.5 \approx 114$. Sampling: systematized random, $k=1.7 \approx 2$. Selection criteria: age $\geq 60$ years old, any gender, without physical disabilities, who agreed to take part in the research. Variables: age, gender, schooling, marital status, occupation, hobbies and leisure time, morbidity, first-level control of chronic-degenerative diseases, hospitalizations, functionality for daily living basic activities (DLBA), functionality for daily living instrumental activities (DLIA), functionality for daily living advanced activities (DLAA), Instruments: Katz scale, Lawton \& Brody index, Siu and Reuben physical scale. Analysis: descriptive statistics and odds ratio $(O R)$ with $95 \%$ of confidence ( $p=0.05$ ). Software: Epi Info $₫ 3.5 .1$ for Windows $(C$ environment.

Results: 114 elderly people, mean age $70.2 \pm 7.3$ years, interval $60-96$ years-old. $54 \%$ was male and $46 \%$ female. $77 \%$ was carriers of diseases. Functional dependence: $15 \%$ basic, $66 \%$ instrumental and $68 \%$ advanced. Associated factors to basic functional dependence: age $>$ national life

1 Centro de Salud Comunitario "General Vicente Guerrero", Cárdenas, Tabasco, México.

2 Sistema Estatal de Investigadores de Tabasco, México.

Correspondencia: Eduardo Fócil-Némiga, katana_as@hotmail.com

Recibido: 30/12/2014 - Revisado: 10/01/2015 - Aceptado: 20/01/2015 
expectancy $\mathrm{OR}=4.3(\mathrm{Cl} 951.45,12.73)$, social role change $\mathrm{OR}=6.32$ (Cl95 1.67, 23.91). Associated factors to instrumental functional dependence: female gender $\mathrm{OR}=4.73$ (CI95 1.97, 11.35). Associated factor to advanced functional dependence: female gender $O R=7.58$ (CI95 2.83, 20.34). Conclusions: The prevalence of dependence for DLBA was low, while for DLIA and DLAA was high. Rehabilitation actions and targeted timely detection programs are required.

Keywords: (MeSH NLM): elderly person, daily activities, disability, functionality, independence.

\section{Introducción}

En 2011 la población mundial alcanzó 650 millones de adultos $\geq 60$ años, estimándose que para 2050 alcanzarán 2,000 millones, de los que $80 \%$ vivirá en países en desarrollo. ${ }^{[1-3]}$ En México, los adultos $\geq$ 60 años representan 7\% de la población y llegarán a 28\% en 2050.4 En Tabasco, para 2010 existían 157,533 adultos mayores, esperándose que en 2030 lleguen a 408,859, pasando de $7.2 \%$ a $16.1 \%{ }^{[4]}$

Las personas 260 años usan más los servicios de salud que cualquier otro grupo de edad ${ }_{3}^{[5]}$ por lo que conocer el envejecimiento fisiológico y patológico es importante, ${ }^{[6]}$ tanto, que la Organización Mundial de la Salud recomienda que todo el equipo de salud tenga formación geriátrica, independientemente de su profesión. ${ }^{[3]}$ Se debe considerar que el adulto mayor cursa padecimientos con características comunes que hacen poco probable la curación y que la rehabilitación sea el enfoque principal. ${ }^{[7]}$

Los padecimientos comunes en el adulto mayor, se engloban bajo el término de "síndromes geriátricos" ${ }^{3 "}{ }^{[8]}$ Los más comunes son: caídas, depresión, fragilidad, inmovilidad, trastornos de la marcha y equilibrio, deterioro cognitivo, incontinencia y dependencia, entre otros. ${ }^{[7-9]}$ La fragilidad en particular, suele englobar al resto.

La fragilidad se caracteriza por disminución de la resistencia y reservas fisiológicas ante situaciones estresantes debido al desgaste de los sistemas, ${ }^{[6]}$ que puede llevar a que un padecimiento produzca discapacidad y dependencia ${ }^{[7]}$.

La discapacidad alcanza niveles sustanciales a partir de los 70 años de edad, cuando el riesgo de experimentar deterioro funcional es mayor. ${ }^{[4]}$ Por ello, es importante incrementar la vida libre de discapacidad, instrumentando estrategias preventivas ${ }^{[4]}$ En este contexto, cobra importancia hacer una valoración geriátrica integral (VGI). ${ }^{[6,7]}$

La VGI está diseñada para cuantificar capacidades y problemas biopsicosociales, permitiendo formular planes de manejo para mejorar diagnósticos, tratamientos, funcionalidad y calidad de vida, disminuyendo ingresos a hospitales. ${ }^{[6,7]}$ En este sentido, la funcionalidad, definida como la capacidad para realizar actividades de la vida diaria (AVD), es útil como marcador del efecto sistémico de una enfermedad, ayudando a definir metas terapéuticas adecuadas y personalizadas. ${ }^{[6,7]}$

La funcionalidad para AVD se divide en tres categorías: actividades básicas de la vida diaria (ABVD), actividades instrumentales de la vida diaria (AIVD) y actividades avanzadas de la vida diaria (AAVD). ${ }^{[6-8]}$ Las ABVD se refieren al cuidado personal, independencia de movimiento-traslado y continencias; ${ }^{[6-}$ ${ }^{8]}$ existen varios instrumentos para su evaluación, como la Escala de Katz, el Índice de Barthel, la Escala de Incapacidad Física de la Cruz Roja y la Escala de Montorio, entre otros. ${ }^{[10]}$ Las AIVD corresponden a la habilidad para manejarse en su entorno, ${ }^{[6-8]} \mathrm{se}$ evalúan a través de un menor número de instrumentos, como el Índice de Lawton \& Brody y el Cuestionario de Actividad Funcional de Pfeffer. ${ }^{[10]}$ Finalmente, las AAVD se refieren al rol social, ${ }^{[6-}$ ${ }^{8]}$ y también existen pocos instrumentos para su evaluación, comola Escala de Salud Funcional de Rosow \& Breslau y la Escala Física de Siu y Reuben. ${ }^{[10]}$

Un envejecimiento exitoso está relacionado con un buen desempeño en todas las AVD. ${ }^{[11-13]}$ Es posible mejorar la vida libre de discapacidades con la evaluación de funcionalidad, pues permite prevenir la aparición de estas. ${ }^{[14-16]}$

Es difícil comparar la bibliografía existente, dados los puntos de corte divergentes para definir a los adultos mayores. ${ }^{[17]}$ Se estima que la prevalencia de dependencia funcional es alta en mayores de 65 años, $24 \%$ para ABVD y $23 \%$ para AIVD, asimismo, se ha observado relación con diversas enfermedades crónicas. ${ }^{[18]}$ En Tabasco, se reportó que en adultos mayores rurales del Municipio de Centro, la prevalencia de dependencia para ABVD es $23 \%$ y para AIVD 86,1\%. ${ }^{[6]}$ Mientras que en adultos mayores urbanos del Municipio de Cárdenas, la dependencia para ABVD es 74\% y para AIVD 92\%. ${ }^{[19]}$

En 2011, el Poblado "General Vicente Guerrero" (C-29), del Municipio de Cárdenas, Tabasco, tenía 3,170 habitantes de los que 194 eran adultos mayores. ${ }^{[20,21]}$ La tasa de mortalidad en ese mismo año fue $0,095 \%$, principalmente por enfermedades cardiovasculares y neumonía. ${ }^{[20,21]}$ Las principales causas de morbilidad entre la población adulta mayor fueron hipertensión arterial, diabetes mellitus, dislipidemia y artrosis, ${ }^{[20,21]}$ lo que hace suponer un gran número de adultos mayores con dependencia funcional, que al momento del estudio no había sido determinado, y que de conocerse, permitiría diseñar e implementar estrategias de prevención y/o rehabilitación, que mejorarían el gasto en materia de salud en la localidad.

En virtud del contexto descrito en los párrafos precedentes, se realizó el presente estudio con el objetivo de evaluar la funcionalidad para las AVD en la población de adultos mayores residentes del Poblado C-29 de Cárdenas, Tabasco, México, en 2011.

\section{Materiales y métodos}

Se realizó una investigación transversal en la que se estudió un universo de 194 adultos mayores residentes del Poblado C-29 de Cárdenas, Tabasco, ${ }^{[20,21]}$ de donde se tomó una muestra probabilística simple $(p=0,23, q=0,77)$ de $n=113,5 \approx 114$, para la que se consideró un nivel de confianza de $95 \%(Z=1,96)$ y un error máximo de $5 \%(\mathrm{~d}=0,05)$. Estos 114 adultos mayores fueron seleccionados mediante muestreo aleatorio sistematizado a intervalos de $\mathrm{k}=1,7 \approx 2$ a partir del censo nominal de la población, incluyéndose sujetos de edad $\geq 60$ años, de cualquier sexo, sin discapacidades físicas, que aceptaron participar proporcionando su consentimiento informado

Se estudiaron las variables: edad, sexo, escolaridad, estado civil, ocupación, actividades de tiempo libre, morbilidad, control de enfermedades crónico-degenerativas, hospitalizaciones y funcionalidad para ABVD, AIVD y AAVD.

Las variables sociodemográficas y sanitarias se obtuvieron mediante interrogatorio directo, cuyas respuestas se registraron en una hoja de recolección de datos. La funcionalidad para ABVD se evaluó con la Escala de Katz, que clasifica al adulto mayor en: 
R.F.S Revista Facultad de Salud

Julio - Diciembre de 2014;6(2): 12-19
Funcionalidad para actividades de la vida diaria en adultos mayores rurales de Cárdenas, Tabasco, México / Eduardo Fócil-Némiga et al. normal, muy levemente incapacitado, levemente incapacitado, moderadamente incapacitado, severamente incapacitado, incapacitado e inválido. La funcionalidad paraAIVD fue evaluada con el Índice de Lawton y Brody, que clasifica al adulto mayor en: independiente, moderadamente dependiente y dependiente. Finalmente, la funcionalidad para AAVD se evaluó con la Escala Física de Siu y Reuben, que clasifica al adulto mayor en: autónomo o no autónomo. ${ }^{[7,10]}$ Estas escalas cuentan con validez de contenido, además de buena fiabilidad interobservadores y estabilidad.

La información fue recopilada por los investigadores, con la colaboración de un grupo de seis voluntarios previamente capacitados para esta labor, quienes visitaron los domicilios de los adultos mayores seleccionados, para realizar el interrogatorio y aplicar los instrumentos señalados anteriormente. Los datos así obtenidos, fueron sistematizados en una base de datos a través de un entorno de captura de información diseñado empleando el software Epilnfo® versión 3.5.1 para entorno Windows ${ }^{\circledR}$, con el que se realizó el análisis estadístico correspondiente.

El análisis de la información se llevó a cabo en dos fases, una descriptiva y otra analítica. En la fase descriptiva, se obtuvieron medidas de frecuencia, de tendencia central y de dispersión, según se trató de variables cualitativas y/o cuantitativas, respectivamente, considerándolas todas descriptoras de la población. Mientras que en la fase analítica se midió la fuerza de asociación entre las variables dependientes e independientes por medio de razones de momios (OR) con 95\% de confianza ( $\mathrm{p} \leq 0,05)$. Para obtener OR se consideró "caso" a las personas que tuvieron algún grado de dependencia en cualquiera de las AVD (ABVD, AIVD y AAVD), y "controles" a los adultos mayores sin dependencia alguna, es decir, totalmente independientes. Estas estadísticas fueron expresadas mediante tablas o gráficos, según fue requerido, para facilitar el análisis y discusión de los resultados, evitando la redundancia.

Este estudio se mantuvo en apego a la Declaración de los Derechos Humanos, ${ }^{[22]}$ el Código de Nüremberg ${ }^{[23]}$ y la Declaración de Helsinki, ${ }^{[24]}$ así como la Ley de Los Derechos de las Personas Adultas Mayores, ${ }^{[25]}$ la Ley General de Salud, ${ }^{[26]}$ y el Reglamento de la Ley General de Salud en Materia de Investigación para la Salud ${ }^{[27]}$; procurando la dignidad, los derechos y el bienestar de la población, en tanto que se trató de una investigación observacional sin riesgo para los participantes, y contó con la autorización de los representantes locales de la Secretaría de Salud.

\section{Resultados}

Se estudió una muestra integrada por 114 adultos mayores residentes del Poblado C-29, Cárdenas, Tabasco, México

\section{Perfil sociodemográfico}

La edad media fue $70,2 \pm 7,3$ años, con una mediana de 70 y una moda de 60, dentro de un intervalo de 60-96. E1 35\% $(n=40)$ fueron de edad mayor a la expectativa de vida nacional ( 74 años). E1 54\% $(\mathrm{n}=61)$ fueron masculinos y $46 \%(\mathrm{n}=53)$ femeninos. Se observaron $73 \%(n=83)$ con escolaridad primaria, $3 \%(n=3)$ secundaria y $1 \%$ $(n=1)$ con licenciatura, asimismo, se encontró $24 \%(n=27)$ analfabetas. $\mathrm{E} 170 \%(\mathrm{n}=80)$ eran de estado civil casado(a), $27 \%(\mathrm{n}=31)$ fue viudo(a), $2 \%(n=2)$ en unión libre y $1 \%(n=1)$ solteros(as). E1 96\% refirió no haber cambiado su rol social con la vejez, las ocupaciones más frecuentes fueron: campesino 46\% y ama de casa $44 \%$; la distribución de frecuencias de estas, se expone en la (Tabla 1).

\section{Tiempo libre y ocio}

E1 96\% (n=109) refirió practicar actividades de tiempo libre y ocio, de los cuales $5 \%(\mathrm{n}=5)$ manifestaron realizar sólo una actividad y $95 \%(n=104)$ refirió llevar a cabo dos. Entre las actividades primarias, la principal actividad practicada fue "convivir con la familia" $(31,2 \%, n=34)$, en segundo lugar "actividades religiosas" $(24,8 \%$, $n=27)$ y en tercer lugar "ver televisión" $(16,5 \%, n=18)$.Entre las actividades secundarias, la principal actividad fue "platicar con los amigos" $(51,9 \%, \mathrm{n}=54)$ y en segundo lugar "convivir con la familia" $(29,8 \%, \mathrm{n}=31)$. La distribución de frecuencias de las actividades de tiempo libre y ocio se presenta en la (Tabla 2).

\section{Morbilidad}

El 77\% $(n=88)$ refirió portar alguna enfermedad. Dentro de esta proporción, $60 \%(\mathrm{n}=53)$ resultó portador de un padecimiento y

Tabla 1. Ocupaciones de la población. Adultos mayores del Poblado C-29, Cárdenas, Tabasco.

\begin{tabular}{|c|c|c|c|c|}
\hline & Ocupaciones & Frecuencia & Porcentaje & $\begin{array}{l}\text { Porcentaje } \\
\text { acumulado }\end{array}$ \\
\hline \multirow[t]{6}{*}{ Sin cambio de rol } & Ama de casa & 50 & 44 & 44 \\
\hline & Campesino & 53 & 46 & 90 \\
\hline & Comerciante & 2 & 2 & 92 \\
\hline & Obrero & 1 & 1 & 93 \\
\hline & Prestador de servicios independiente & 3 & 3 & 96 \\
\hline & Profesionista & 1 & 1 & 97 \\
\hline \multirow[t]{2}{*}{ Cambio de rol } & Jubilado & 2 & 2 & 99 \\
\hline & Pensionado & 2 & 2 & 100 \\
\hline
\end{tabular}

Fuente: Encuesta aplicada. 
Tabla 2. Actividades de tiempo libre y ocio. Adultos mayores del Poblado C-29, Cárdenas, Tabasco.

\begin{tabular}{|c|c|c|c|c|}
\hline & Actividades & Frecuencia & Porcentaje & $\begin{array}{l}\text { Porcentaje } \\
\text { acumulado }\end{array}$ \\
\hline Primarias & $\begin{array}{l}\text { Actividades religiosas } \\
\text { Convivir con la familia } \\
\text { Crianza de animales } \\
\text { Jardinería } \\
\text { Leer } \\
\text { Manualidades } \\
\text { Otras } \\
\text { Platicar con los amigos } \\
\text { Salir a caminar } \\
\text { Tocar instrumento musical } \\
\text { Ver televisión }\end{array}$ & $\begin{array}{l}27 \\
34 \\
3 \\
4 \\
4 \\
2 \\
3 \\
5 \\
8 \\
1 \\
18\end{array}$ & $\begin{array}{l}24,8 \\
31,2 \\
2,8 \\
3,7 \\
3,7 \\
1,8 \\
2,8 \\
4,6 \\
7,3 \\
0,9 \\
16,5\end{array}$ & $\begin{array}{l}24,8 \\
56,0 \\
58,7 \\
62,4 \\
66,1 \\
67,9 \\
70,6 \\
75,2 \\
82,6 \\
83,5 \\
100,0\end{array}$ \\
\hline Secundarias & $\begin{array}{l}\text { Actividades religiosas } \\
\text { Convivir con la familia } \\
\text { Cuidar a los nietos } \\
\text { Otras } \\
\text { Platicar con los amigos } \\
\text { Realizar negocios con conocidos } \\
\text { Salir a caminar } \\
\text { Ver televisión }\end{array}$ & $\begin{array}{c}2 \\
31 \\
1 \\
7 \\
54 \\
1 \\
5 \\
3\end{array}$ & $\begin{array}{c}1,9 \\
29,8 \\
1,0 \\
6,7 \\
51,9 \\
1,0 \\
4,8 \\
2,9\end{array}$ & $\begin{array}{c}1,9 \\
31,7 \\
32,7 \\
39,4 \\
91,3 \\
92,3 \\
97,1 \\
100,0\end{array}$ \\
\hline
\end{tabular}

Fuente: Encuesta aplicada.

$40 \%(n=35)$ de dos. En esta misma proporción, el 69.3\% $(n=61)$ se encontró en control médico al momento del estudio.

La Diabetes Mellitus fue la enfermedad primaria más frecuente $(34,1 \%, n=30)$ y en segundo lugar la Hipertensión Arterial
Esencial $(27,3 \%, \mathrm{n}=24)$. Mientras que entre la morbilidad concomitante, la Hipertensión Arterial Esencial fue la más frecuente $(48,6 \%, n=17)$. Las causas de morbilidad de la población, se exponen en la (Tabla 3 ).

Tabla 3. Causas de morbilidad, Adultos mayores del Poblado C-29, Cárdenas, Tabasco.

\begin{tabular}{|c|c|c|c|c|}
\hline & Morbilidad & Frecuencia & Porcentaje & $\begin{array}{l}\text { Porcentaje } \\
\text { acumulado }\end{array}$ \\
\hline Primaria & $\begin{array}{l}\text { Deterioro cognitivo } \\
\text { Diabetes Mellitus } \\
\text { Dislipidemia } \\
\text { Enfermedad cerebro vascular } \\
\text { Enfermedad de Parkinson } \\
\text { Fractura(s) } \\
\text { Hiperplasia prostática } \\
\text { Hipertensión arterial esencial } \\
\text { Osteoartritis } \\
\text { Otras }\end{array}$ & $\begin{array}{c}3 \\
30 \\
6 \\
2 \\
2 \\
1 \\
2 \\
24 \\
5 \\
13\end{array}$ & $\begin{array}{l}3,4 \\
34,1 \\
6,8 \\
2,3 \\
2,3 \\
1,1 \\
2,3 \\
27,3 \\
5,7 \\
14,8\end{array}$ & $\begin{array}{c}3,4 \\
37,5 \\
44,3 \\
46,6 \\
48,9 \\
50,0 \\
52,3 \\
79,5 \\
85,2 \\
100\end{array}$ \\
\hline Concomitante & $\begin{array}{l}\text { Diabetes Mellitus } \\
\text { Dislipidemia } \\
\text { Hipertensión arterial esencial } \\
\text { Osteoartritis } \\
\text { Otras }\end{array}$ & $\begin{array}{c}4 \\
5 \\
17 \\
1 \\
8\end{array}$ & $\begin{array}{l}11,4 \\
14,3 \\
48,6 \\
2,9 \\
22,9\end{array}$ & $\begin{array}{l}11,4 \\
25,7 \\
74,3 \\
77,1 \\
100,0\end{array}$ \\
\hline
\end{tabular}

Fuente: Encuesta aplicada. 
R.F.S Revista Facultad de Salud

Julio - Diciembre de 2014;6(2): 12-19
Funcionalidad para actividades de la vida diaria en adultos mayores rurales de Cárdenas, Tabasco, México / Eduardo Fócil-Némiga et al.
Entre los 114 adultos mayores, 16 (14\%) refirieron antecedentes de ingreso a hospital durante el último año previo al estudio, de los cuales $94 \%(n=15)$ manifestó sólo una hospitalización y 6\% $(n=1)$ refirió dos

\section{Funcionalidad para la vida diaria}

Se observó que $15 \%(n=17)$ de los adultos mayores resultaron con algún nivel de dependencia funcional básica, siendo los grados más frecuentes el "muy levemente incapacitado" y el "incapacitado", de los que se presentaron 5 casos (4\%) de cada uno. En cuanto a la funcionalidad para AIVD, se observó que 66\% $(n=75)$ resultaron con algún nivel de dependencia funcional instrumental, siendo el grado "moderadamente dependiente" el más frecuente del que se presentaron 52 casos (46\%). Finalmente, con respecto a la funcionalidad para AAVD, se observó que $68 \%$ $(n=78)$ resultaron con dependencia funcional avanzada. La funcionalidad para AVD de la población estudiada, en sus tres niveles, se presenta en la (Tabla 4 ).

\section{Factores asociados a dependencia funcional}

Al buscar factores asociados a dependencia funcional básica, se encontró asociación estadísticamente significativa con "edad superior a la expectativa de vida nacional" $\mathrm{OR}=4,3\left(\mathrm{IC}_{95} 1,45\right.$, $12,73)$, y con "cambio de rol social" $\mathrm{OR}=6,32\left(\mathrm{IC}_{95} 1,67,23,91\right)$. Por otra parte, al examinar factores asociados a dependencia funcional instrumental, se encontró asociación estadísticamente significativa con "sexo femenino" $\mathrm{OR}=4,73\left(\mathrm{IC}_{95} 1,97,11,35\right)$. Finalmente, al explorar factores asociados a dependencia funcional avanzada, se encontró asociación estadísticamente significativa con "sexo femenino" $\mathrm{OR}=7,58\left(\mathrm{IC}_{95} 2,83,20,34\right)$. Los factores asociados y no a dependencia funcional para los distintos niveles de AVD, se exponen en la (Tabla 5).

\section{Discusión}

Primeramente, es pertinente señalar las limitaciones y sesgos de esta investigación, para considerar sus resultados objetivamente. En cuanto a limitaciones, el estudio se realizó en una única comunidad, por lo que sus resultados no se pueden generalizar. Respecto a los sesgos, por ser un estudio observacional puede haber sesgos derivados de la falta de control sobre el medio,en tanto que los errores de aleatorización (tipo I) y sistematización (tipo II) fueron controlados al estudiarse una muestra probabilísticaobtenida aleatoriamente. Asimismo, los criterios de selección fueron amplios, por lo quela población fue heterogénea, lo que puede tener influencia sobre las mediciones. En este orden de ideas, el exceso de roles atribuidos al género femenino presentes en la Escala de Lawton \& Brody, ${ }^{[10]}$ pueden ocasionar falsos positivos en el género masculino, asimismo, los criterios de evaluación para una misma escala pueden variar de un estudio a otro por falta de consenso. Considerando lo anterior, es menester analizarlos resultados de este estudio a la luz de los reportes consultados.

En cuanto al perfil demográfico, la media de edad fue similar a la informada por De La Fuente-Bacelis y cols. ${ }^{[6]}$ y por ZavalaGonzález y cols. ${ }^{[19]}$. Respecto al sexo, los resultados contrastan con los de Zavala-González y cols. ${ }^{[19]}$ y Barrantes-Monge y cols., ${ }^{[18]}$ donde el sexo femenino predominó.Asimismo, el estado civil unido fue el más frecuente en este estudio, lo que concuerda con lo reportado por Guerrero-Pérez y cols. ${ }^{[28]}$ pero difiere con lo informado por Zavala-González y cols. ${ }^{[19]}$. En este orden de ideas, en esta serie se observó que la escolaridad fue baja en 99\%, de forma similar a lo informado por Zavala-Gonzáles y cols., ${ }^{[19]}$ y cercano a lo publicado por Guerrero-Pérez y cols. ${ }^{[28]}$ con respecto al analfabetismo. Finalmente, se observó que las ocupaciones más frecuentes fueron campesino y ama de casa, lo que concuerda con los resultados de Zavala González y cols. ${ }^{[19]}$ así como Guerrero-Pérez y cols. ${ }^{[28]}$. Si

Tabla 4. Funcionalidad para AVD, Adultos mayores del Poblado C-29, Cárdenas, Tabasco.

\begin{tabular}{|c|c|c|c|c|}
\hline & Estado funcional & Frecuencia & Porcentaje & $\begin{array}{l}\text { Porcentaje } \\
\text { acumulado }\end{array}$ \\
\hline \multirow[t]{6}{*}{ Funcionalidad para ABVD } & Incapacitado & 5 & 4 & 4 \\
\hline & Levemente incapacitado & 4 & 4 & 8 \\
\hline & Moderadamente incapacitado & 1 & 1 & 9 \\
\hline & Muy levemente incapacitado & 5 & 4 & 13 \\
\hline & Normal & 97 & 85 & 98 \\
\hline & Severamente incapacitado & 2 & 2 & 100 \\
\hline \multirow[t]{3}{*}{ Funcionalidad para AIVD } & Dependiente & 23 & 20 & 20 \\
\hline & Independiente & 39 & 34 & 54 \\
\hline & Moderadamente dependiente & 52 & 46 & 100 \\
\hline \multirow{2}{*}{ Funcionalidad para AAVD } & Autónomo & 36 & 32 & 32 \\
\hline & Dependiente & 78 & 68 & 100 \\
\hline
\end{tabular}

Fuente: Escala de Katz, Índice de Lawton \& Brody y Escala Física de Siu \& Reuben aplicadas. 
Tabla 5. Factores asociados y no a dependencia funcional para AVD, Adultos mayores del Poblado C-29, Cárdenas, Tabasco.

\begin{tabular}{|c|c|c|c|c|c|c|c|c|c|}
\hline \multirow{3}{*}{$\begin{array}{l}\text { Variables de exposición } \\
\text { Edad superior a la expectativa de vida nacional }\end{array}$} & \multicolumn{3}{|c|}{$\begin{array}{l}\text { Dependencia } \\
\text { para ABVD }\end{array}$} & \multicolumn{3}{|c|}{$\begin{array}{l}\text { Dependencia } \\
\text { para AIVD }\end{array}$} & \multicolumn{3}{|c|}{$\begin{array}{l}\text { Dependencia } \\
\text { para AAVD }\end{array}$} \\
\hline & \multirow{2}{*}{$\begin{array}{l}\text { OR } \\
4,3\end{array}$} & \multicolumn{2}{|c|}{$\mathrm{IC}_{95}$} & \multirow{2}{*}{$\begin{array}{c}\text { OR } \\
2,35\end{array}$} & \multicolumn{2}{|c|}{$\mathrm{IC}_{95}$} & \multirow{2}{*}{$\begin{array}{c}\text { OR } \\
1,98\end{array}$} & \multicolumn{2}{|c|}{$1 \mathrm{C}_{95}$} \\
\hline & & 1,45 & 12,73 & & 0,98 & 5,64 & & 0,82 & 4,77 \\
\hline Sexo femenino & 1,03 & 0,37 & 2,88 & 4,73 & 1,97 & 11,35 & 7,58 & 2,83 & 20,34 \\
\hline Baja escolaridad (secundaria o menor) & - & - & - & - & - & - & - & - & - \\
\hline $\begin{array}{l}\text { Estado civil no unido (soltero, separado, } \\
\text { divorciado, viudo) }\end{array}$ & 1,34 & 0,45 & 3,99 & 2,57 & 1,00 & 6,61 & 1,75 & 0,70 & 4,37 \\
\hline Cambio de rol social (jubilado/pensionado) & 6,32 & 1,67 & 23,91 & - & - & - & - & - & - \\
\hline Práctica de actividades de TL y ocio & - & - & - & - & - & - & - & - & - \\
\hline Portador de enfermedad & 5,56 & 0,7 & 44,07 & 1,94 & 0,79 & 4,74 & 2,29 & 0,93 & 5,64 \\
\hline Hipertensión Arterial Esencial & 0,97 & 0,33 & 2,84 & 2,04 & 0,87 & 4,80 & 1,71 & 0,73 & 4,05 \\
\hline Diabetes Mellitus & 1,81 & 0,63 & 5,25 & 1,36 & 0,57 & 3,25 & 2,19 & 0,85 & 5,66 \\
\hline Control de enfermedades & 0,36 & 0,12 & 1,09 & 1,19 & 0,45 & 3,15 & 0,91 & 0,32 & 2,53 \\
\hline Antecedentes de hospitalización & 1,38 & 0,35 & 5,48 & 1,67 & 0,50 & 5,56 & 1,45 & 0,43 & 4,87 \\
\hline
\end{tabular}

Fuente: Elaboración propia.

bien, existen algunas diferencias puntuales respecto a los autores locales consultados, se puede asumir que en general, la población estudiada tiene las características comunes de la población del Estado de Tabasco a la que pertenece.

Respecto a las actividades de tiempo libre y ocio, se observó que las más comúnmente referidas por la población fueron "convivir con la familia" y "actividades religiosas", lo que coincide con lo informado por Zavala González y cols. ${ }^{[19]}$ y Guerrero-Pérez y cols. ${ }^{[28]}$. Estas similitudes pueden deberse a la idiosincrasia compartida de los sujetos, quienes pertenecen al Estado de Tabasco, México.

En lo tocante a la morbilidad, el padecimiento más frecuente fue Hipertensión Arterial Esencial, concordando con lo referido por Barrantes-Monge y cols. ${ }^{[18]}$. Éste resultado pudiera atribuirse al componente biológico de la enfermedad.

En cuanto a las AVD, en esta serie 15\% de los sujetos mostró dependencia para $A B V D$, lo que contrasta con el $74 \%$ informado por Zavala González y cols., ${ }^{[19]}$ que puede atribuirse a que estos autores sólo estudiaron usuarios de servicios de salud, probablemente afectados por enfermedades que derivanen mayor prevalencia de dependencia; en este sentido, los resultados obtenidos son más cercanos a los reportados por Arias Merino y cols., ${ }^{[17]}$ De La Fuente-Bacelis y cols., ${ }^{[6]}$ y Barrantes Monge y cols., ${ }^{[18]}$ con resultados de $9,6 \%, 22,8 \%$ y $24 \%$, respectivamente. Respecto a las AIVD, $66 \%$ de la población resultó con dependencia, cifra inferior a los hallazgos de De La Fuente-Bacelis y cols., ${ }^{[6]}$ y de Zavala-González y cols., 19 quienes reportaron $86,1 \%$ y $92 \%$ respectivamente, y al mismo tiempo, mayor al $31.5 \%$ observado por Arias-Merino y cols ${ }^{[17]}$ estas diferencias pueden atribuirse a los tipos de poblaciones comparadas, pues se trata de una comunidad rural, una urbana y usuarios de un asilo. No se encontraron referencias para comparar los resultados sobre funcionalidad para AAVD, por lo que no se puede afirmar que el $68 \%$ de dependencia observado sea elevado o bajo.
Finalmente, en cuanto a factores asociados a los distintos niveles de dependencia funcional, con respecto a dependencia para $\mathrm{ABVD}$, la asociación de riesgo con superar la expectativa de vida nacional, coincide con lo informado por Barrantes-Monge y cols. ${ }^{[18]}$ y por Zavala-González, ${ }^{[19]}$ mientras que la asociación de riesgo con el estatus de jubilado/pensionado no había sido aludido por otros autores consultados. En lo tocante a dependencia para AIVD, la asociación con el sexo femenino coincide con lo reportado por AriasMerino, ${ }^{[17]}$ sin embargo, no se encontró este tipo de asociación con Hipertensión Arterial Esencial y Diabetes Mellitus, lo que difiere con lo expuesto por Barrantes-Monge y cols., ${ }^{[18]}$ en tanto que tampoco se encontró la asociación protectora con estado civil no unido referida por Zavala-González ${ }^{[19]}$. Finalmente, en lo tocante a dependencia para AAVD, se observó asociación de riesgo con el sexo femenino, pero no se encontraron referencias que hicieran alusión a este hallazgo.

\section{Conclusiones}

El perfil sociodemográfico de los adultos mayores del Poblado C-29 de Cárdenas, Tabasco, México, corresponde a un sujeto de género indistinto con una edad promedio de 70.2 años, de escolaridad baja, que se dedica al campo o las labores del hogar, de estado civil unido, casado, sin cambio de rol social. Estos adultos mayores realizaban actividades de tiempo libre y ocio, siendo las más frecuentes son "convivir con la familia" y participar en "actividades religiosas". La Hipertensión Arterial Esencial fue el padecimiento más frecuente, lo que concuerda con las estadísticas estatales.

La prevalencia de dependencia para ABVD es baja, mientras que más de la mitad de la población resultó con algún grado de dependencia para AIVD o AAVD. 
R.F.S Revista Facultad de Salud

Julio - Diciembre de 2014;6(2): 12-19
Funcionalidad para actividades de la vida diaria en adultos mayores rurales de Cárdenas, Tabasco, México /Eduardo Fócil-Némiga et al.
En el Poblado C-29 existe riesgo de dependencia en ABVD al superar la expectativa de vida nacional y con el cambio de rol social al pasar a ser jubilado o pensionado. Mientras que el sexo femenino expone a la población a dependencia para AIVD y AAVD, por lo que se debe hacer detección oportuna en este grupo

Se requieren estudios posteriores, con inclusión de otras variables como somatización, depresión, funcionalidad y dinámica familiar, entre otras, así como análisis multivariado para diferenciar posibles variables confusoras, con el objetivo de obtener más y mejores resultados. Asimismo, se requieren estudios multicéntricos para obtener resultados generalizables aplicables a regiones de diversa magnitud

Es recomendable implementar la aplicación rutinaria de la Escala de Katz, el Índice de Lawton \& Brody y la Escala Física de Siu $\&$ Reuben como herramientas para la detección oportuna de deterioros funcionales para $\mathrm{ABVD}, \mathrm{AIVD}$ y $\mathrm{AAVD}$

\section{Aclaraciones}

Contribuciones de autoría: EFN y MAZG concibieron y diseñaron la investigación. EFN aportó el material de estudio además de coordinar y supervisar la recolección de la información. MAZG analizó e interpretó los datos, y redactó el artículo. EFN y MAZG realizaron la revisión crítica del artículo y aprobaron su versión final. Financiamiento: Ninguno. Conflictos de intereses: Ninguno. Agradecimientos: A Reyna del Carmen Hernández González, Andrés Alejandro Fortunat Fócil, Juan Lorenzo Hernández González, Eduardo Rodríguez Peláez, y Mario Uriel Pérez Zamudio, por su colaboración en la recolección de información. A los revisores anónimos asignados a este manuscrito, por su contribución a la mejora de la calidad del mismo. Presentaciones previas: Presentado en la modalidad de cartel durante el XXI Foro Sur de Investigación en Salud, celebrado en la Ciudad de Villahermosa, Centro, Tabasco, México, del 11 al 13 de junio de 2014.

\section{Referencias}

1. Fondo de Población de las Naciones Unidades (UNFPA). Estado de la población mundial. 7000 millones de personas: su mundo sus posibilidades [monografía en Internet]. México: UNFPA, 2011 [consultado, noviembre 2013]. Disponible en http://www.unfpa.org.mx/ publicaciones/ SP-SWOP2011.pdf

2. Organización Mundial de la Salud. Envejecimiento y ciclo de vida [monografía en Internet]. Suiza: WHO/ OMS, 2011 [consultado, diciembre 2013]. Disponible en http://www.who.int/features/ factfiles/ageing/es/

3. Organización Mundial de la Salud. Envejecimiento. Datos y cifras [monografía en Internet]. Suiza: WHO/ OMS, 2011 [consultado, noviembre 2013]. Disponible en http://www. who.int/features/factfiles/ageing/ ageing facts/es/index.html

4. Zúñiga E, Vega D. Consejo Nacional de Población. El Envejecimiento de la población de México. Reto del siglo XXI. 1 edición. México D.F. México: CONAPO. 2004:22,73,11,36.

5. Olaiz-Fernández G, Rivera-Dommarco J, Shamah-Levy T, Rojas R, Villalpando-Hernández S, Hernández-Ávila M, Sepúlveda-Amor J. Encuesta Nacional de Salud y
Nutrición 2006. Cuernavaca, México: Instituto Nacional de Salud Pública. 2006:45.

6. De la Fuente-Bacelis Teresa J, Quevedo-Tejero EC, Jiménez-Sastré A, Zavala-González MA. Funcionalidad para las actividades de la vida diaria en el adulto mayor de zonas rurales. Arch Medicina Fam. 2010;12(1):1-4.

7. Duthie Edmund, Katz Paul R, Malone Michael L. Practice of Geriatrics, 4th ed. Philadelphia, Pennsylvania. E.U.A.: Elsevier Saunders. 2007:5,41-52,255-70.

8. García-Zenón T, López-Guzmán JA, Villalobos-Silva JA, d'Hyver de las Deses C. Prevalencia de síndromes geriátricos en ancianos hospitalizados. Med Int Mex. $2006 ; 22(5): 369-374$

9. Gallardo L, d'Hyver de las Deses C. Prevalencia de síndromes geriátricos en una residencia de mujeres de edad avanzada. Med Int Mex. $2011 ; 27(1): 23-28$

10. De Lucas-Vaquero MV Compilación de técnicas para una evaluación multidimensional en vejez. Un protocolo de evaluación [monografía en Internet]. Madrid, España: UGR, 2003 [consultado, diciembre 2013]. Disponible en http://www.ugr.es/ master/ master/publicaciones/compilacion.tecnica.jpg

11. Elsawy B, Higgins K. E. The Geriatric assessment. Am Fam Physician 2011 ;83(1):48-56.

12. Lowry KA, Vallejo AN, Studenski SA. Successful aging as a continuum of functional independence: Lessons from physical disability models of aging. Aging and disease. 2012;3(1):5-15M.

13. Bowling $C B$, Fonarow GC, Patel K, Zhang Y, Feller MA, Sui $X$, Blair SN, Alagiakrishnan K, Aban IB, Love TE, Allman RM, Ahmed A. Impairment of activities of daily living and incident heart failure in community-dwelling older adults. Eur J Heart Fail first published online April 4, 2012 doi:10.1093/eurjhf/hfs034 [consultado, noviembre 2013]. Disponible en http://www.ncbi. nlm.nih.gov/pubmed/22492539

14. Hashimoto $S$, Kawado $M$, Yamada H, Seko R, Murakami Y, Hayashi M, Kato M, Noda T, Ojima T, Nagai M, Tsuji I. Gains in Disability-Free Life Expectancy From Elimination of Diseases and Injuries in Japan. J Epidemiol. 2012;22(3):199-204 [consultado, noviembre 2013]. Disponible en https://www.jstage.jst.go.jp/ article/jea/22/3/22_JE20110112/_pdf

15. Stineman MG, Henry-Sánchez JT, Kurichi JE, Pan Q, Xie $D$, Saliba D, Zhang $Z$, Streim JE. Staging activity limitation and participation restriction in elderly community-dwelling persons according to difficulties in self-care and domestic life functioning. Am J Phys Med Rehabil. 2012;91(2):126-140.

16. Arnau A, Espaulella J, Serrarols M, Canudas J, Formiga $F$, Ferrer $M$. Factores asociados al estado funcional en personas de 75 o más años de edad no dependientes. Gac Sanit. 2012. doi:10.1016/i.gaceta.2011.09.035

17. Arias-Merino ED, Mendoza-Ruvalcaba NM, Ortiz GG, Velázquez-Brizuela IE, Meda-Lara RM, Cueva-Contreras J. Physical function and associated factors in communitydwelling elderly people in Jalisco, México. Arch Gerontol Geriatr. 2012;54(3):e271-8. 
18. Barrantes-Monge M, García-Mayo EJ, Gutiérrez-Robledo $L M$, Miguel-Jaimes A. Dependencia funcional y enfermedades crónicas en ancianos mexicanos. Salud Publica Mex. $2007 ; 49$ (Suppl4):S459-66.

19. Zavala-González MA, Domínguez-Sosa G. Funcionalidad para la vida diaria en adultos mayores. Rev Med Inst Mex Seguro Soc. $2011 ; 49(6): 585-590$.

20. Secretaría de Salud. Diagnóstico de Salud. Poblado "General Vicente Guerrero" C-29, Cárdenas Tabasco: Secretaría de Salud 2011.

21. Secretaría de Salud. Concentrado de Tarjetas Censales Familiares. Poblado "General Vicente Guerrero" C-29, Cárdenas Tabasco: Secretaría de Salud 2011.

22. ONU. Declaración Universal de los Derechos Humanos [monografía en Internet]. E.U.A: ONU, 2012 [consultado, noviembre 2013]. Disponible en http:// www.un.org/es/documents/udhr/

23. Observatori de Bioetica i Dret Parc Cientific de Barcelona. Código de Nüremberg [monografía en Internet]. España: UB, 2012 [consultado, noviembre 2013]. Disponible en http://www.pcb.ub.edu/bioeticaidret/archivos/norm/CodigoNuremberg.pdf
24. Asamblea Médica Mundial. Declaración de Helsinki [monografía en Internet]. México: UNAM, 2012 [consultado, noviembre 2013]. Disponible en http:// www.inb.unam. mx/bioetica/documentos/declaracion_ helsinki.pdf

25. Instituto Nacional de las Personas Adultas Mayores. Ley de las Personas Adultas Mayores (última reforma DOF24-06-2009). México D.F., México: Diario Oficial de la Federación. 2009.

26. Secretaría de Salud. Ley General de Salud (última reforma DOF 30-09-2013). México D.F., México: Diario Oficial de la Federación. 2013.

27. Secretaría de Salud. Reglamento de la Ley General de Salud en Materia de Investigación para la Salud (última reforma DOF 03-02-1983). México D.F., México: Diario Oficial de la Federación. 1983.

28. Guerrero-Pérez R, Quevedo-Tejero EC, García-Rochín R, Zavala-González MA. Perfil gerontológico del adulto mayor en Tabasco, México. Rev Salud Pública (Bogotá) $2012 ; 14(1): 88-101$. 